\title{
Highly sensitive fiber Bragg grating refractive index sensors
}

\author{
Wei Liang, Yanyi Huang, ${ }^{\text {a) }}$ Yong Xu, Reginald K. Lee, and Amnon Yariv \\ Department of Applied Physics, California Institute of Technology, Pasadena, California 91125
}

(Received 31 January 2005; accepted 16 March 2005; published online 8 April 2005)

\begin{abstract}
We combine fiber Bragg grating (FBG) technology with a wet chemical etch-erosion procedure and demonstrate two types of refractive index sensors using single-mode optical fibers. The first index sensor device is an etch-eroded single FBG with a radius of $3 \mu \mathrm{m}$, which is used to measure the indices of four different liquids. The second index sensor device is an etch-eroded fiber Fabry-Pérot interferometer (FFPI) with a radius of $\sim 1.5 \mu \mathrm{m}$ and is used to measure the refractive indices of isopropyl alcohol solutions of different concentrations. Due to its narrower resonance spectral feature, the FFPI sensor has a higher sensitivity than the FBG sensor and can detect an index variation of $1.4 \times 10^{-5}$. Since we can measure the reflection signal, these two types of sensors can be fabricated at the end of a fiber and used as point sensors. (C) 2005 American Institute of Physics. [DOI: $10.1063 / 1.1904716]$
\end{abstract}

Since the early 1990s, fiber Bragg grating (FBG) sensors have been intensively developed due to their many desirable advantages such as the small size, absolute measurement capability, immunity to electromagnetic interference, wavelength multiplexing, and distributed sensing possibilities. ${ }^{1-5}$ Thus far, the FBG sensors' capability to measure physical quantities such as the temperature, strain, pressure, etc., has been studied extensively. ${ }^{2-8}$ However, the use of FBG sensors for detection of environmental refractive index change has not been fully explored. Refractive index sensing is important for biological and chemical applications since a number of substances can be detected through measurements of the refractive index. ${ }^{2-4,8-13}$ For normal FBGs, removal of the fiber cladding is required to increase the evanescent field interaction with the surrounding environment. This concept has been demonstrated using D-shaped fiber and sidepolished fiber. $9,11,12$ In both cases, the strength and durability of the sensor were greatly reduced. Special fiber was also needed, which would raise the costs and limit the possible applications. Long-period fiber gratings have also been demonstrated to have high sensitivity to the refractive index of the ambient media, ${ }^{2,3,13-15}$ however, their multiple resonance peaks and broad (typically tens of nanometers) transmission resonance features limit the measurement accuracy and their multiplexing capabilities. ${ }^{9}$ In addition, the relatively long length of the grating limits their application as point sensor devices.

In this letter, we first demonstrate a single etch-eroded FBG sensor using standard single-mode telecommunication fiber (Corning SMF-28). Fiber Fabry-Pérot interferometers (FFPIs) have also been widely used as sensors. ${ }^{2,3,16,17}$ Compared to a single FBG, the FFPI sensors possess narrower resonance peaks and are more desirable for high accuracy wavelength measurement. ${ }^{2,3,9,18}$ To that end we propose and demonstrate an etch-eroded FFPI sensor formed by two FBGs. The FFPI sensor is used to measure the refractive index of isopropyl alcohol (IPA) solutions of different concentrations, exhibiting the capability of detecting very small index variations of the ambient medium.

\footnotetext{
a) Author to whom correspondence should be addressed; electronic mail: yanyi@caltech.edu
}

Fig. 1(a) shows the schematic of the FBG sensor and the FFPI sensor. For a FBG sensor, the center wavelength of the reflection band, $\lambda_{B}$, is given by ${ }^{2}$

$$
\lambda_{B}=2 \cdot n_{\mathrm{eff}} \Lambda,
$$

where $\Lambda$ is the period of the Bragg grating determined by the phase mask, and $n_{\text {eff }}$ is the effective modal refractive index. The index of the ambient material, $n_{\text {ambient }}$, can be determined from the Bragg wavelength $\lambda_{B}$, given the core index $n_{\mathrm{co}}$ and the radius $a$ of the etched fiber. ${ }^{19}$

For the etch-eroded FFPI formed by two FBGs, we assume that the effective cavity length $L_{\mathrm{FP}}$ is a constant, and that the wavelength of a resonance mode, $\lambda_{R}$, must satisfy ${ }^{19}$

$$
m \lambda_{R} / 2=n_{\mathrm{eff}} L_{\mathrm{FP}}
$$

where $m$ is the modal number. When $n_{\text {ambient }}$ is changed, the effective index is shifted by $\Delta n_{\text {eff }}$ and the resonance wave-
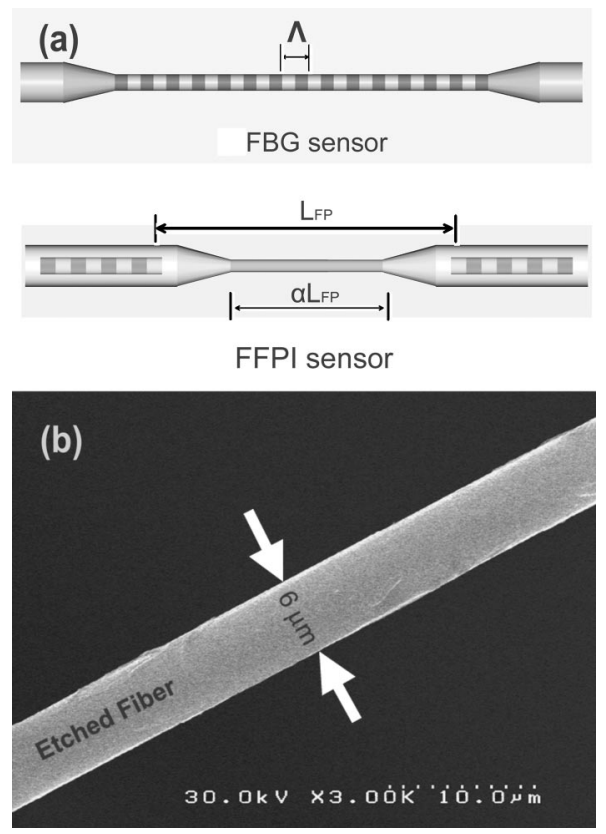

FIG. 1. (a) Schematic of a single fiber Bragg grating (FBG) sensor and a fiber Fabry-Pérot interferometer (FFPI) sensor. (b) Scanning electron microscope (SEM) image of an etch-eroded fiber. 

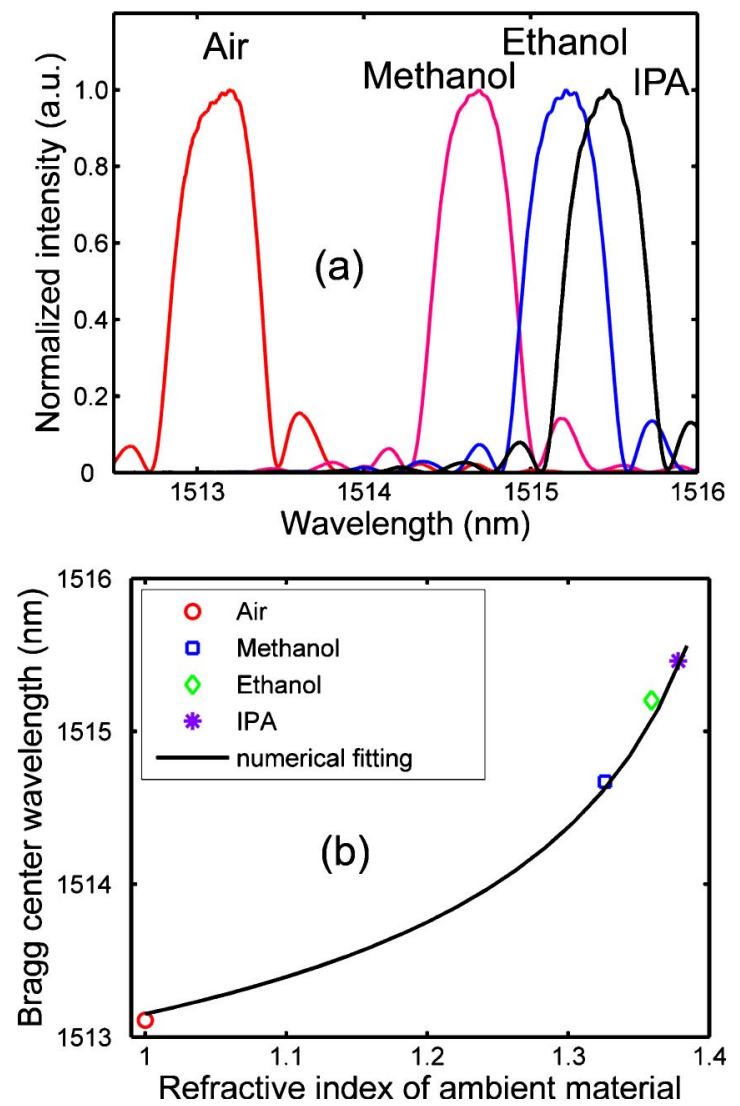

FIG. 2. (a) (Color online) Reflection spectra of an etch-eroded single fiber Bragg grating (FBG) sensor immersed in different liquids. (b) The Bragg center wavelength as a function of the refractive index of ambient material. The markers are Bragg center wavelengths extracted from (a), the fitting curve is calculated using Eq. (1) with parameters $n_{\mathrm{co}}=1.454$ and $a$ $=2.975 \mu \mathrm{m}$.

length of the $m$ th mode is shifted by $\Delta \lambda_{R}$. From Eq. (2), we can derive

$$
\Delta \lambda_{R} / \lambda_{R}=\alpha \cdot \Delta n_{\text {eff }} / n_{\text {eff }},
$$

where $\alpha$ is the fraction of the optical length of the FFPI that is etch eroded [see Fig. 1(a)].

In our experiment, the FBG is written in a hydrogenloaded Corning SMF-28 fiber using a $\mathrm{KrF}$ excimer laser (248 $\mathrm{nm})$ and an amplitude phase mask $(\Lambda=524.58 \mathrm{~nm})$. The length of the FBG is $2.5 \mathrm{~mm}$. The wet-chemical etching setup is similar to the one shown in Ref. 20. We first etch the fiber with a 52\% hydrofluoric (HF) acid solution for $30 \mathrm{~min}$ to a fiber diameter below $15 \mu \mathrm{m}$. Since the etching speed of the Ge-doped core is faster than that of the silica cladding, ${ }^{21}$ the etching solution is then replaced with a $13 \%$ HF solution to slow down the etching process. The diameter of the final etch-eroded fiber is controlled by monitoring the transmission loss in situ. ${ }^{20}$ Figure 1(b) shows a scanning electron microscope (SEM) image of an etch-eroded fiber, which has a very uniform thickness.

We then put the FBG sensor in different liquids and measure the reflection spectra by scanning the wavelength using a tunable laser (Agilent 8164A). The reflection spectra for the FBG sensor in air $(n=1.00)$, methanol $(n=1.326)$, ethanol $(n=1.359)$, and isopropyl alcohol (IPA) $(n=1.378)$ are shown in Fig. 2(a). As expected, the reflection spectrum redshifts as the ambient refractive index increases. The Bragg wavelength as a function of the ambient refractive index is Downloaded 14 Dec 2005 to 131.215.225.171. Redistribution subje
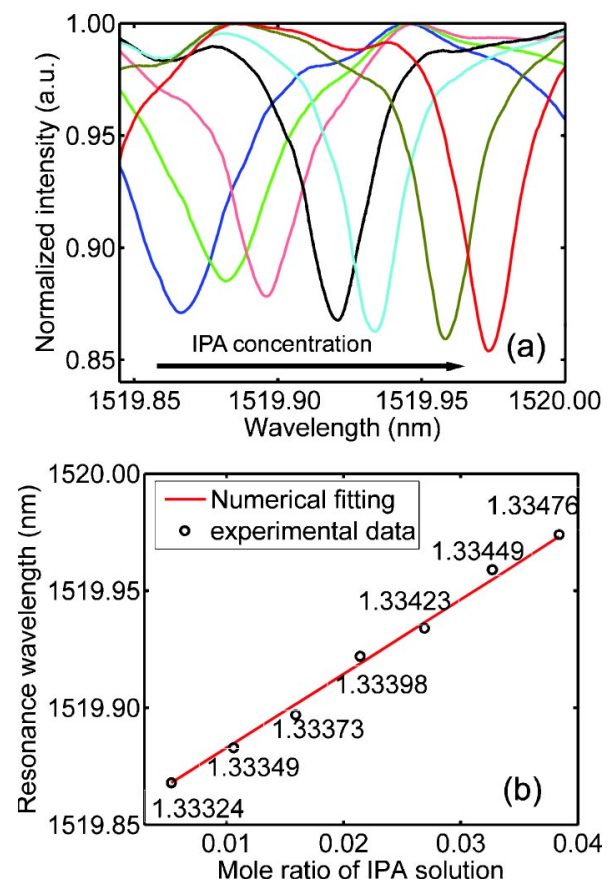

FIG. 3. (a) (Color online) Reflection spectra of the resonance mode of a fiber Fabry-Pérot interferometer (FFPI) sensor in isopropyl alcohol (IPA) solution with different concentrations. (b) The resonance wavelength as a function of the mole ratio of IPA solution. The circles are resonance wavelengths extracted from (a), the numerical fitting is obtained using Eq. (3) with parameters $n_{\mathrm{co}}=1.454, a=1.475 \mu \mathrm{m}$ and $\alpha=0.9$.

shown in Fig. 2(b) and fit to Eq. (1) with $\Lambda$ (524.58 nm), $n_{\text {co }}$ (1.454), $n_{\text {ambient }}$ and an appropriate choice of the fiber radius $a$. The effective modal index $n_{\text {eff }}$ in Eq. (1) is obtained by numerically solving the $\mathrm{HE}_{11}$ modal equation of a step-index cylindrical waveguide. ${ }^{19}$ The fitting parameter $a=2.975 \mu \mathrm{m}$ agrees well with the measured value of $3.0 \mu \mathrm{m}$ as shown in Fig. 1(b).

The FFPI sensor is formed by two FBGs written on a hydrogen-loaded Corning SMF-28 fiber. The distance between the two FBGs is chosen to be $\sim 5 \mathrm{~mm}$ so that there are less than three resonance modes in the band gap of the FBG. To eliminate the influence of the ambient media on the FBG band gap in the measurements, we mask the fiber to selectively etch the region between the two gratings without affecting the gratings.

Compared to the FBG sensor, the FFPI sensor should have a higher refractive index resolution due to its sharper resonance feature. To demonstrate this, we use it to detect the variation of the refractive indices of IPA-water solutions with different IPA concentrations, which allows arbitrarily small changes of index. Figure 3(a) shows the redshift of a resonance mode due to the increase of the concentration of the IPA-water solution. In Fig. 3(b) we plot the resonance wavelength as a function of the mole ratio of the IPA in solution. The refractive indices of the IPA solution shown in Fig. 3(b) are estimated based on the mole ratio of each component, i.e., $\quad n_{\text {ambient }}=\left[\kappa \cdot n_{\mathrm{IPA}}^{2}+(1-\kappa) \cdot n_{\text {water }}^{2}\right]^{1 / 2}, \quad$ where $n_{\text {water }}$ $=1.333$ is the refractive index of water, $n_{\mathrm{IPA}}$ is the refractive index of pure IPA, and $\kappa$ is the mole ratio of IPA in the solution. The effective modal index $n_{\text {eff }}$ can then be calculated based on $n_{\text {ambient }}, n_{\text {co }}(1.454)$, and the fiber radius $a .^{19}$ We fit the resonance wavelengths to Eq. (3) as shown in Fig. 3 (b), where the parameters are $\alpha=0.9$ (based on the sensor geometry), $\lambda_{R}=1519.868 \mathrm{~nm}, n_{\mathrm{eff}}=1.42021$ (based on the to AlP license or copyright, see http://apl.aip.org/apl/copyright.jsp 
first experimental point), and $a=1.475 \mu \mathrm{m}$. The fiber radius used in the fit agrees well with the measured radius of $1.5 \mu \mathrm{m}$. Using the slope of the fitted curve, we can define the sensor responsivity as $\Delta \lambda / \Delta n=71.2 \mathrm{~nm}$. This number is an order of magnitude higher than the one demonstrated in Ref. 9. The sensitivity of the sensor is limited by the stability of the whole measuring system. In our experiment, the fluctuation of the resonance wavelength is around $1 \mathrm{pm}$, which indicates the sensor has the potential to detect an index variation of $1.4 \times 10^{-5}$. It is also interesting to compare the performance of the FFPI sensor with that of the FBG sensor. As can be measured from Figs. 2(a) and Fig. 3(a), the resonance spectra of the FFPI sensor have an approximate full width (at half extinction) of $0.025 \mathrm{~nm}$ as compared to $0.5 \mathrm{~nm}$ (full width at half maximum) for the FBG sensor. This means the resolution of the FFPI sensor is enhanced by 20 times over that of the FBG sensor.

Beside the high sensitivity, these etch-eroded devices have some additional advantages. The maximum tensile stress in a bent fiber of radius $a$ is at the surface of the fiber and is given by ${ }^{23}$

$$
\sigma \propto E \cdot a / R
$$

where $E$ is the Young's modulus of the fiber and $R$ is the radius of the curvature. Thus, with the same bending curvature, the induced tensile stress in the fiber decreases with the reduction of the fiber radius. Since the radius of the etcheroded fiber sensor is on the order of $1 \mu \mathrm{m}$, it has a higher flexibility compared to a fiber sensor with a larger diameter, and can be bent or coiled without being broken. ${ }^{23,24}$ This characteristic ensures the durability of the sensor devices. In addition, since both the FBG and the FFPI sensors can be used in reflection mode, they can be fabricated at the end of a fiber and used as point detectors. Finally, by coating the etch-eroded fiber surface with a thin layer of binding reagents, these sensors can be functionalized to detect specific biological and/or chemical agents with high sensitivity.

In conclusion, we have combined FBG and wetchemical silica etching techniques to demonstrate a FBG refractive index sensor and a FFPI refractive index sensor in standard SMF-28 optical fiber. The FFPI sensor has a responsivity of $\Delta \lambda / \Delta n=71.2 \mathrm{~nm}$ and displays the capability of detecting an index variation of $1.4 \times 10^{-5}$. These highly flexible sensors add the function of refractive index sensing to the established repertoire of FBG sensing techniques, and can be applied to many fields, including biochemical sensing and environmental monitoring.

The authors thank Will Green, John M. Choi and Joyce Poon for helpful discussions. This work is supported by the National Science Foundation (DMR-0120967) and the Defense Advanced Research Projects Agency (N00014-04-10094).

${ }^{1}$ K. O. Hill, Y. Fujii, D. C. Johnson, and B. S. Kawasaki, Appl. Phys. Lett. 32, 647 (1978).

${ }^{2}$ F. T. S. Yu, S. Yin, Fiber Optic Sensors (Dekker, New York, 2002), Chaps. 2 and 4 and p. 124.

${ }^{3}$ Y. J. Rao, Opt. Lasers Eng. 31, 297 (1999).

${ }^{4}$ A. D. Kersey, M. A. Davis, H. J. Patrick, M. Le Blanc, K. P. Koo, C. G. Askins, M. A. Putnam, and E. J. Friebele, J. Lightwave Technol. 15, 1442 (1997).

${ }^{5}$ I. Bennion, A. R. Williams, L. Zhang, K. Sugden, and N. J. Doran, Opt. Quantum Electron. 28, 93 (1996).

${ }^{6}$ X. Shu, K. Chisholm, I. Felmeri, K. Sugden A. Gillooly, L. Zhang, and I. Bennion, Appl. Phys. Lett. 83, 3003 (2003).

${ }^{7}$ D. C. Betz, L. Staudigel, M. N. Trutzel, and M. Kehlenbach, Struct. Health Monitoring 2, 145 (2003).

${ }^{8}$ Y. J. Rao, D. J. Webb, D. A. Jackson, L. Zhang, and I. Bennion, J. Lightwave Technol. 15, 779 (1997).

${ }^{9}$ K. Zhou, X. Chen, L. Zhang, and I. Bennion, Electron. Lett. 40, 232 (2004).

${ }^{10}$ C. Barian, I. R. Matias, I. Romeo, J. Garrido, and M. Laguna, Appl. Phys. Lett. 77, 2274 (2000).

${ }^{11}$ W. Ecke, K. Usheck, V. Hagemann, R. Mueller, and R. Willsch, Proc. SPIE 3555, 457 (1998).

${ }^{12}$ G. Meltz, S. J. Hewlett, and J. D. Love, Proc. SPIE 2836, 342 (1996).

${ }^{13}$ V. Bhatia and A. M. Vengsarkar, Opt. Lett. 21, 692 (1996).

${ }^{14}$ X. Shu and D. X. Huang, Opt. Commun. 171, 65 (1999).

${ }^{15}$ X. Shu, X. L. Zhang and I. Bennion, J. Lightwave Technol. 20, 255 (2002).

${ }^{16}$ T. G. Giallorenzi, J. A. Bucaro, A. Dandridge, G. H. Sigel, J. H. Cole, S. C. Rashleigh, and R. G. Priest, IEEE J. Quantum Electron. 18, 626 (1982).

${ }^{17}$ A. Wang, H. Xiao, J. Wang, Z. Wang, W. Zhao, and R. G. May, J. Lightwave Technol. 19, 1495 (2001).

${ }^{18}$ D. Monzon-Hernandez, J. Villatoro, D. Talavera, and D. Luna-Moreno, Appl. Opt. 43, 1216 (2004).

${ }^{19}$ A. Yariv, Optical Electronics in Modern Communications. 5th ed. (Oxford University Press, New York, 1997), pp. 80-88 and p. 129.

${ }^{20}$ J. P. Laine, B. E. Little, and H. A. Haus, IEEE Photonics Technol. Lett. 11, 1429 (1999).

${ }^{21}$ D. J. Markos, B. L. Ipson, K. H. Smith, S. M. Schultz, R. H. Selfridge, T. D. Monte, R. B. Dyott, and G. Miller, Appl. Opt. 42, 7121 (2003).

${ }^{22}$ C. Gaffney and C. K. Chau, Am. J. Phys. 69, 821 (2001).

${ }^{23}$ M. J. Matthewson, C. R. Kurkjian, and S. T. Gulati, J. Am. Ceram. Soc. 69, 815 (1986).

${ }^{24}$ L. Tong, R. R. Gattass, J. B. Ashcom, S. He, J. Lou, M. Shen, I. Maxwell, and E. Mazur, Nature (London) 426, 816 (2003). 